\title{
A qualitative examination of the current management of opioid use disorder and barriers to prescribing buprenorphine in a Canadian emergency department
}

David Wiercigroch ${ }^{1 *}$, Patricia Hoyeck ${ }^{1}$, Hasan Sheikh ${ }^{2,3}$ and Jennifer Hulme $e^{2,3}$

\begin{abstract}
Background: Emergency departments (EDs) across Canada are increasingly prescribing buprenorphine for opioid use disorder (OUD). The objective of this study was to identify the current knowledge, attitudes, and behaviours of ED physicians on the management of OUD in the ED, including barriers and facilitators to prescribing buprenorphine.

Methods: We purposefully selected emergency physicians from one ED in Toronto which had recently received education on OUD management and had a new addiction medicine follow-up clinic, to participate in semi-structured interviews. We used semi-structured interviews to explore experiences with patients with OUD, conceptions of role of the ED in addressing OUD, and specifically ask about perceptions and experience on using buprenorphine for opioid withdrawal. Our analysis was informed by constructivist grounded theory to help uncover contextualized social processes and focus on what people do and why they do it. Two researchers independently coded transcripts using an iterative constant comparative and interpretative approach.

Results: Results fell broadly into facilitators and barriers. Generally, management of OUD in the ED varied significantly. Physician-level facilitators to treating opioid withdrawal with buprenorphine included: knowledge about OUD an7d buprenorphine, positive experiences with substitution therapy in the past, and the presence of physician champions. Systems-level facilitators included timely access to follow-up care and pre-printed order sets. Barriers included provider inexperience, lack of feedback on treatment effectiveness, limited time to counsel patients, and pressure to discharge patients quickly. Additional barriers included concerns about precipitating withdrawal, prescribing a chronic medication in acute care, and patient attitudes.

Conclusion: This study describes barriers and facilitators to addressing OUD and prescribing buprenorphine in a Canadian ED. These findings suggest a role for additional provider education, involvement of allied health professionals in counseling, and mentorship by physician champions in the department.
\end{abstract}

Keywords: Buprenorphine, Opioid withdrawal, Suboxone

* Correspondence: d.wiercigroch@mail.utoronto.ca

${ }^{1}$ Faculty of Medicine, University of Toronto, 1 King's College Circle, Toronto,

Canada

Full list of author information is available at the end of the article

(c) The Author(s). 2021 Open Access This article is licensed under a Creative Commons Attribution 4.0 International License, which permits use, sharing, adaptation, distribution and reproduction in any medium or format, as long as you give appropriate credit to the original author(s) and the source, provide a link to the Creative Commons licence, and indicate if changes were made. The images or other third party material in this article are included in the article's Creative Commons licence, unless indicated otherwise in a credit line to the material. If material is not included in the article's Creative Commons licence and your intended use is not permitted by statutory regulation or exceeds the permitted use, you will need to obtain permission directly from the copyright holder. To view a copy of this licence, visit http://creativecommons.org/licenses/by/4.0/. The Creative Commons Public Domain Dedication waiver (http://creativecommons.org/publicdomain/zero/1.0/) applies to the data made available in this article, unless otherwise stated in a credit line to the data. 


\section{Background}

Opioid Use Disorder (OUD) is a problematic pattern of opioid use that results in impairment or distress of clinical significance [1]. OUD is a cause of significant and increasing harm, with 14,700 opioid-related deaths in Canada between January 2016 and September 2019 [2]. During this time period, 1 in 4 patients seen in EDs for opioid-related poisonings were admitted, resulting in 19, 490 hospitalizations [2]. Since opioid-users frequently present to the ED in withdrawal, following an overdose, or with complications of injection drug use, it is an important opportunity to initiate treatment for OUD.

A recent Canadian clinical guideline recommends the use of the opioid agonist medication buprenorphinenaloxone (trade name Suboxone), from here on referred to as buprenorphine, as first-line treatment for OUD [3]. Buprenorphine offers advantages over methadone, including a ceiling effect which limits the abuse potential and risk of diversion, reduced stigma, and milder withdrawal symptoms [4,5]. Buprenorphine is increasingly accessible in Ontario after being included on the Ontario Drug Benefit formulary [6]. Buprenorphine induction in the ED has been shown to be a feasible and effective approach to begin medication for addiction treatment [79]. A randomized clinical trial found that ED-initiated buprenorphine significantly increased engagement in addiction treatment and decreased use of inpatient addiction services compared to referral or brief interventions in the ED [7]. Retention on buprenorphine treatment has been shown to reduce ED utilization [10].

Widespread adoption of new practices takes time and has particularly lagged in the treatment of substance use disorders [11]. Previous studies have examined attitudes of primary care providers towards prescribing buprenorphine [12-15] as well as those of emergency physicians [16-18]. Yet there is limited literature on attitudes, knowledge and behaviours surrounding OUD and initiating buprenorphine in Canadian EDs [16]. We sought to explore Canadian emergency physician attitudes and experiences in order to inform communications and quality improvement strategies during the opioid crisis and help support patients and providers alike as buprenorphine prescribing expands across Canada.

To achieve this objective, we used grounded theory, a systematic qualitative methodology which is used to generate theories about social processes, experiences, and interactions $[19,20]$. A constructivist approach to grounded theory recognizes that there can be multiple perspectives of reality which are shaped by outcomes, experiences, and interpretation within a changing context [21]. Constructivism also draws on researchers' expertise and experiences to inform data collection and analysis. Constructivist grounded theory is increasingly used to understand health care processes, particularly in nursing research [22].

\section{Methods}

The objective of this study was to assess the attitudes, motivation, experiences, and practices in the treatment of OUD in an ED in Toronto, Ontario. Our qualitative study design was informed by constructivist grounded theory to allow us to generate theory rooted in the data [20], while remaining reflexive during data collection. The research team consisted of two medical trainees with health policy backgrounds (DW, PH) and two academic emergency and addictions physicians $(\mathrm{JH}$ and HS). This approach thus allowed us to acknowledge our assumptions throughout, and that data analysis is a coconstructed process $[21,23]$.

\section{Sampling}

We purposefully sampled ED physicians from one urban academic teaching hospital in Toronto, Ontario. This department was chosen as a department 'in transition' which had some exposure to OUD management: all physicians had received educational rounds on OUD management, an introduction to an opioid withdrawal order set, and the hospital had established a rapid-access addictions clinic for follow-up. We randomly invited half of the physicians from that group of eighty physicians to participate. We performed a simple randomization process using a random number generator to minimize selection bias. We limited invitations to half of the department's physicians to ensure that all interviews could be completed within the study period.

\section{Data collection}

We drafted and field tested a flexible semi-structured interview guide with topic experts, and modified interview questions to ensure developing categories were explored.

The guide consisted of open-ended questions about clinicians' experiences with patients with opioid use disorder. We prompted if needed on their conceptions of the role of the ED in addressing opioid addiction, preventing future overdoses, and counselling around opioid use, treatment options, and harm reduction. The final portion elicited clinicians' experiences and perceived barriers to prescribing buprenorphine, their training, interest, and resources required to facilitate prescribing buprenorphine. The interview guide is available for reference [see Additional file 1].

The first author (DW) conducted semi-structured interviews in person and over the phone based on participant preference between July and September 2018. Data collection and analysis continued until we reached theoretical thematic saturation after 19 participant interviews. Interviews ranged from fifteen to forty-five minutes in length. We recorded and transcribed the interviews verbatim, using pseudonyms during transcription to ensure 
anonymity. We obtained informed verbal consent from all participants and maintained confidentiality. The University Health Network Research Ethics Board approved the protocol as part of a quality improvement project to improve the care of patients with OUD.

\section{Data analysis}

Constructivist grounded theory guided a step-wise approach to simultaneous data collection and analysis [21]. The first four transcripts were reviewed independently by DW, JH and HS to develop familiarity with the data and consensus on a broad coding framework. Two reviewers (DW, PH) independently organized and coded the transcripts through multiple readings to identify meaningful patterns, using an iterative constant comparative and interpretative approach. DW and $\mathrm{PH}$ reviewed the themes to ensure representativeness of the data and to reach a consensus on discordant views. A third reviewer $(\mathrm{JH})$ reviewed and triangulated the thematic analysis with the

Table 1 Participant quotes supporting variability in OUD management of OUD

\section{Variable management of opioid withdrawal}

i. "I think it's a much more nuanced decision-making process to dea with withdrawal than with a patient who is acutely intoxicated and not breathing, someone you can use naloxone with as an intervention." (Physician 12)

ii. "I have a lot less experience treating people with opioid withdrawal than with an overdose and that I find can be extremely frustrating." (Physician 19)

iii. "There are some physicians who just simply take more time in terms of providing patient education and some who do less of that because of time restraints. There's also variability in terms of physician interest in the subject matter. There's variability in terms of what physicians feel should be done about these issues and what they feel their role is in the whole realm of addictions." (Physician 4)

iv. "I think more and more in terms of habits, people are comfortable using [buprenorphine]." (Physician 12)

v. "There are a variety of different medications that you can use to deal with these people when they're leaving. There's low doses of clonidine, NSAIDs and laxatives. Before you discharge them, you want to sure that people are comfortable enough to leave. It depends on what kind of symptoms they're having, whether or how quickly you're able to discharge them." (Physician 13)

vi. "Clonidine is something that people have been using for a long time and using acetaminophen and naproxen to treat other symptoms of withdrawal. I think those things people are comfortable with and have been using for quite a while. I suspect there's probably some people that give opiates and some people that give a lot of benzodiazepines, but I don't. Some people, they give nothing and say no one dies of opiate withdrawal. I think it probably varies a lot." (Physician 19)

vii. "As a group we've decided we would not like to prescribe narcotics. I think there's still a large number of other individuals that take other stances. Oh well, it's Friday. Oh well, this person's a nuisance. I'm just going to write whatever it is and give it to them and get them out of the department. I think that still goes on." (Physician 8)

viii. "Opioid withdrawal is not terribly successful. We don't have the same medications as we do for alcohol withdrawal and I don't think they're effective." (Physician 15)

ix. "We never would have given them naloxone kits. We never would have given the buprenorphine. So all that is pretty new." (Physician 13) two main reviewers. Data analysis was organized using Dedoose software (version 8.0.35, SocioCultural Research Consultants LLC, Los Angeles, CA).

\section{Results}

Nineteen physicians participated in the study. Participants were predominantly male $(n=12 ; 63 \%)$ Almost half of participants $(n=9)$ were in their first five years of practice, five were mid-career having practiced for 6-20 years, and five had been in practice for over 20 years. Almost half $(n=9 ; 47 \%)$ were family physicians with additional training in emergency medicine (CCFP-EM), six were Royal College trained in emergency medicine (FRCP), and four were family physicians (CCFP).

Predominant concepts and themes are summarized here, with supportive quotes outlined in Tables 1, 2, and 3. Results were grouped under broad categories of barriers and facilitators to providing evidence-based treatment of opioid use disorder in the ED. Overall, we found wide variation in the current approaches to treating OUD in the ED. Barriers to treating OUD and prescribing buprenorphine in the ED included physician reports of limited knowledge and experience, inadequate nursing support, safety concerns, patient factors, and logistical challenges. Facilitators to managing opioid withdrawal included the presence of physician champions and positive experiences with opioid agonist therapy, as well as system-level factors such as the availability of order sets and timely access to follow-up for patients.

\section{Variable management of opioid withdrawal}

Participants varied in their approach to managing opioid withdrawal and opioid use disorder. Several participants indicated that opioid withdrawal management was frustrating and required nuanced decisionmaking (Table 1.1i, ii). Many physicians recognized that their approach to medical management and counselling practices likely differed from their colleagues (1.1iii). While some physicians counseled patients extensively on treatment options, harm reduction practices and available resources, others stated that they counselled patients minimally.

Some physicians offered and initiated buprenorphine (1.1iv) while others used non-agonist treatments to target symptoms (e.g. clonidine, non-steroidal anti-inflammatories, anti-diarrheals, benzodiazepines) (1.1v, vi). Participants recognized that opioid medications might be inappropriately prescribed in some cases to patients in opioid withdrawal (1.1 vii). One participant did not feel that there were effective medications for opioid withdrawal (1.1viii). Nevertheless, most physicians interviewed were familiar with buprenorphine and pointed out that its use in the department was increasing, although still in its early stages (1.1ix). 
Table 2 Participant quotes supporting facilitators to treating withdrawal and initiating buprenorphine

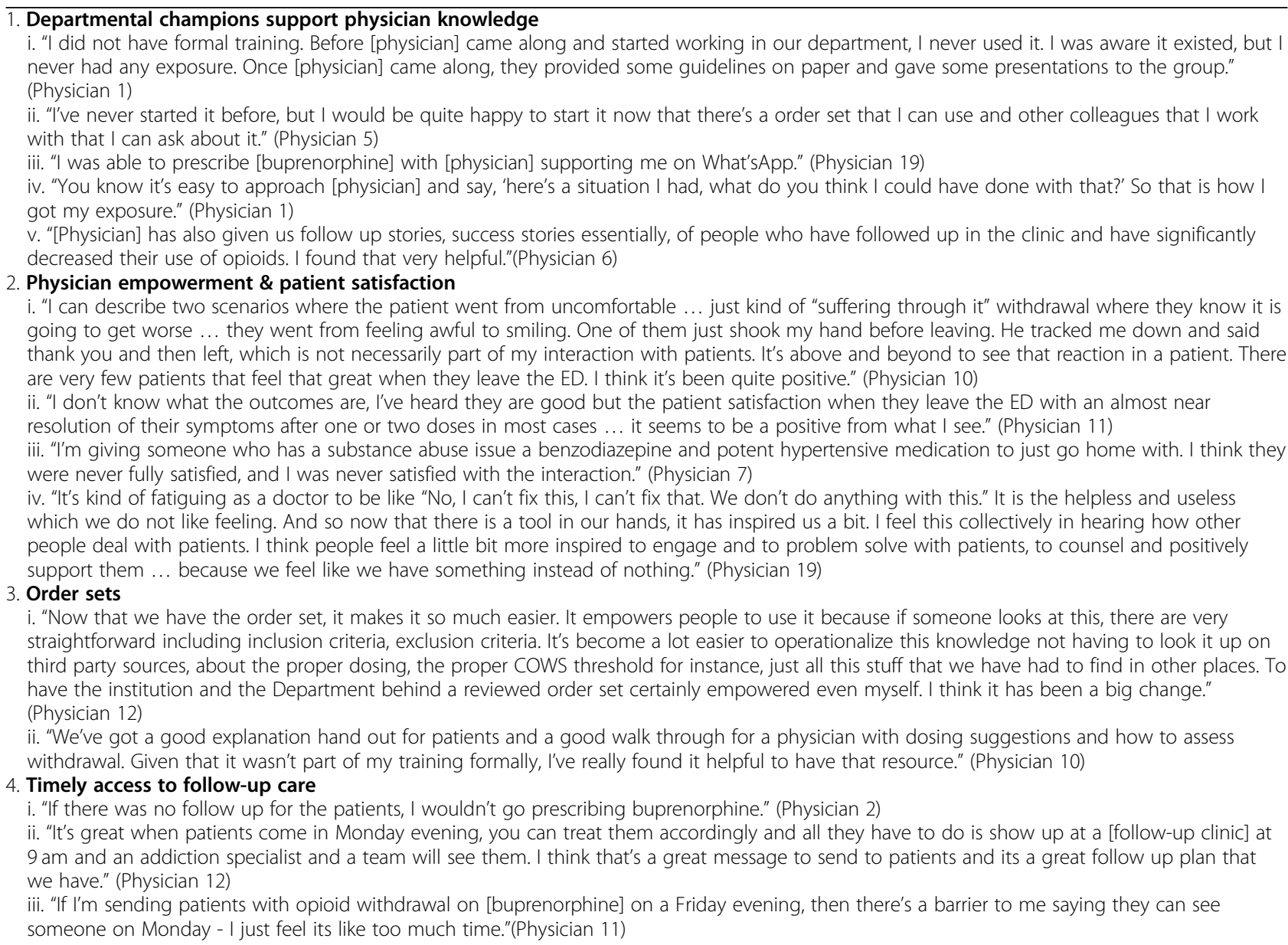

\section{Facilitators to evidence-based treatment of opioid withdrawal in the ED \\ Departmental champions and support}

Physicians frequently stated that their colleagues helped them change their approach to managing opioid withdrawal in the ED including prescribing buprenorphine. These physician champions educated their colleagues about opioid withdrawal and the benefits of buprenorphine (Table 2.1i). Physicians frequently referred to mentorship and informal discussions about their patient cases with more experienced colleagues as important factors in increasing their comfort with prescribing buprenorphine (2.1ii, iii, iv). Some also felt that hearing success stories of patients who had decreased their opioid use after presenting to the ED in withdrawal served as an important motivator to learn more (2.1v).

\section{Physician Empowerment \& Patient Satisfaction}

A few physicians who had initiated buprenorphine in the ED gave accounts of significant patient satisfaction due to resolution of symptoms (Table $2.2 \mathrm{i}$, ii),. These accounts contrasted sjarply with reported experiences using other non-agonist medications which did not resolve symptoms effectively and raised safety concerns (2.2iii). Most physicians with experiences prescribing buprenorphine felt it empowered them to provide a meaningful intervention to a patient population for whom they had no effective options previously (2.2iv).

\section{Protocols and order sets}

Most ED physicians indicated that having an order set for buprenorphine induction was helpful in the management of opioid withdrawal. The order set prompted physicians to consider prescribing buprenorphine, provided inclusion and exclusion criteria as well as dosing recommendations. Physicians felt that the order set instilled greater confidence and was well-integrated into their workflow (Table 2.3i, ii). The order set is available for reference [see Additional file 2]. 
Table 3 Participant quotes supporting barriers to treating withdrawal and initiating buprenorphine

\section{Lack of knowledge \& experience among the care team}

i. "The ones who are a little bit trickier is sometimes we see people who present with the gastrointestinal side effects. So, lots of diarrhea and stomach cramps. Those are cases where I find sometimes it is a bit harder to tease out and they may be in people who are misusing their prescribed opiates." (Physician 19)

ii. "I think generally people are not comfortable and don't think that prescribing [buprenorphine] is a good idea in an emergency setting because of concern about precipitating withdrawal." (Physician 4)

iii. "The only thing I worry is that, and I have not looked this up myself, is long term safety. So do we know that this is a good long term intervention or the possible harm that we just haven't encountered yet because we don't have the numbers or the research for it that we're going to pick up in five or 10 years." (Physician 16)

iv. "When I ordered it the nurses were almost all like "what's a COWS". They had never heard of Cows the first time I did it and that was after it had been going on for a little while." (Physician 19)

v. "[Buprenorphine] was supposed to kind of package it all together and so even though we have suboxone readily available there's a lot of gray area where it can't be prescribed. And so then we still don't know what to do with those patients." (Physician 9)

vi. "Sometimes it was other co-diagnoses like mental health disorders that made it challenging for them to organize the new information. And sometimes it was just someone who had been to our hospital like over 150 times with overdoses and he's just so unwell that he isn't able to be open to a new idea." (Physician 10)

vii. most of the time my patients arrive, and it is too short a time frame since their last consumption. By the time we get to the point of having a conversation about taking a prescription of that stuff home quite often they are ready to bolt and they're not prepared to consider it." (Physician 1)

viii. "If they are on methadone, it's not a medication we can really give them." (Physician 10)

2. Patient disposition and behavior

i. "I would say it's challenging: rarely an easy interaction, rarely do they understand the consequences of what happened especially if they've been given Narcan because they often don't have a recollection of the events and don't believe what you're telling them." (Physician 16)

ii. "The challenge, the biggest point of conflict I find is often when someone should probably stay but they want to go home. We don't think it's safe for them to leave. It can be really challenging trying to decide: are they competent to make the decision? Do you restrain them and keep them here?"(Physician 19)

iii. "When I refuse to give them [opioids], the first step that they usually go for is "well get somebody else here" ... that they want to see another doctor. I will not give them that. Then they'll say, "well I'm going to complain to the hospital" and ... "I'm going to report you to the college" and I'll say "be my guest". Then they'll start calling me names and swearing at me and using foul language and that's usually when I get security to escort them out." (Physician 2)

iv. "Some people have told me "Look I'm not interested. I've tried it and I don't like it." (Physician 10)

3. Logistical constraints

i. "When people come in intoxicated or in withdrawal, they take up a bed for a long time... And so our gut reaction is "we have a busy emergency department and now you're being demanding of my time and a pain". It takes time with repeated doses in the emergency department." (Physician 19)

ii. "I don't think that we can be doing a good job at providing all that counseling ourselves. So given the volumes of patients that we see typically, the length of the interaction with each patient, I don't think its feasible." (Physician 14)

iii. "They are very high users of the emergency department, and you can put in a little bit of time upfront and decrease their burden on the department." (Physician 11)

iv. "I think ED overcrowding plays a big role in our management of patients in withdrawal ... the issue of physical space is not without significance." (Physician 19)

v. "Some people sometimes are reluctant to talk about them in the hallway if they're in a hallway stretcher because they don't want everybody else around to hear what their problems are." (Physician 4)

4. Initiating a chronic medication in an acute care setting

i. "[Buprenorphine] is really a chronic medication that people will start and continue taking indefinitely. And you know it's a bit of a philosophical change. I don't think most ER doctors like the idea of prescribing medications that are used long-term." (Physician 15)

ii. "I think that its akin to saying like do you start blood pressure medications in the emergency department. The patients who are hypertensive. And I have to say that a lot of my colleagues do not because we don't have a way of following up on those prescriptions." (Physician 14) iii. "Follow up, repeated prescriptions and ... titration of dose would be the other thing. We don't really aim to provide that in the ER for pretty much any other condition mostly because we don't intend on seeing you ever again. That's our philosophy." (Physician 15)

iv. "[Buprenorphine] is not necessarily a medication that I thought would be an emergent use but the more that I see that it can kind of quench some of the significant opioid withdrawal symptoms ... I think once we kind of heard that as a group there was more of an acceptance of using this medication in the emergency department." (Physician 6)

v. "The patients that I have prescribed it for, I've had a good response from them initially whether those patients continue to do other follow up and things like that I have no idea." (Physician 18)

vi. "I think the biggest thing is the follow up is unclear always. I mean we initiate these things and we don't know whether our initiation of them is having an actual positive effect. It's not really a barrier to our starting it but I guess it's more if we are starting it, is it definitely helpful or not?" (Physician 3)

\section{Timely access to follow-up care}

Physicians identified timely follow-up as an important factor in their willingness to initiate buprenorphine in the ED (Table 2.4i). Physicians felt that the follow-up should be available the next day, though some were comfortable if patients could access it within the next few days (2.4ii). Limited follow-up during the weekend reduced their comfort with prescribing buprenorphine in some cases (2.4iii).

\section{Barriers to evidence-based treatment of opioid withdrawal} Lack of experience and confidence among the care team Most physicians still felt inexperienced with managing opioid withdrawal, with some concerned about missing subtle presentations (Table 3.1i) and the risk of precipitating withdrawal when initiating buprenorphine therapy (Table 3.1ii). Others noted uncertainty about the long- 
term safety of buprenorphine as a barrier to prescribing (Table 3.1iii). Many physicians hesitated to use the opioid withdrawal order set as the nursing staff lacked familiarity with buprenorphine and the Clinical Opiate Withdrawal Scale (COWS) (Table 3.1iv).

Some physicians who were interested in prescribing buprenorphine experienced challenges when they encountered more complex cases (3.1.v). Factors such as medical or psychiatric comorbidities (3.1vi) or a patient's lack of interest in treatment (3.1vii) presented additional challenges. Some physicians encountered difficulties with buprenorphine induction for patients who used methadone or had recently used other opioids (3.1vii, viii).

\section{Negative patient interactions}

In some cases, physicians had experienced negative interactions with patients which affected their motivation to counsel and treat OUD in the ED. Physicians spoke of cases of aggressive behaviours which made for stressful encounters (3.2i). Tensions between patient and physician arose in cases where the patient wanted to leave after an overdose but the physician felt it was unsafe (3.2ii) or when the patient requested an opioid medication which the physician did not believe was indicated 3.2iii). These adversarial interactions limited the establishment of rapport and presented a barrier to further counselling and discussion of buprenorphine therapy. In some cases, patients declined due to previous poor experiences with the medication (3.2iv).

\section{Logistical constraints}

Physicians commonly cited time constraints as a barrier to treating opioid withdrawal in the ED. Some physicians identified waiting for the patient to reach an appropriate level of withdrawal and subsequent monitoring upon initiating the medication as barriers (Table 3.3i). Concerns around time requirements for proper counselling were common (3.3ii). Some physicians disagreed that time constraints were a barrier to the management of withdrawal and rather focused on the potential to reduce future visits by intervening (3.3iii).

The physical environment of the ED was cited as an important barrier to comprehensive management of opioid withdrawal, including overcrowding (3.3iv), "hallway medicine" and a lack of privacy (3.3v). Physicians felt that both time and physical constraints made extensive counselling difficult to take on in the ED.

\section{Initiating a chronic medication in an acute care setting}

Some physicians did not feel that prescribing a longterm medication in an ED setting was appropriate (Table 3.4i) with comparisons to hypertension and hyperlipidemia management (3.4ii). The need for medication titration, uncertainty of immediate follow-up and longterm management were reported (3.4iii). Some perspectives changed when they considered the potential of this medication to effectively address opioid withdrawal in the acute setting (3.4iv).

Furthermore, many physicians felt uncertain about the effectiveness of their intervention in managing opioid withdrawal. Those that referred patients to the outpatient follow-up clinic identified that they had no feedback on whether the patient had attended (3.4v). Most felt that it would be helpful to know if patients were attending (3.4vi).

\section{Discussion}

This is a qualitative study describing barriers and facilitators to addressing OUD and prescribing buprenorphine in an urban Canadian ED setting. The Canadian guidelines recommend buprenorphine as first-line for management of OUD [3]. Our findings suggest highly variable approaches to managing opioid withdrawal and the need for a targeted knowledge translation strategy to address common barriers and shift ED culture to one that celebrates treatment of acute presentations to affect long-term outcomes. In Canada, there are no regulatory barriers or exemption programs needed in order to prescribe buprenorphine [24].

Most physicians reported limited or no experience prescribing buprenorphine and expressed feelings of dissatisfaction and frustration with their current interventions for OUD in the ED. Similar perspectives have been reported in a recent survey of ED clinicians in a tertiary center in the United States [18]. A key difference though was that a minority of the interviewees in that study were in favor of ED-initiated buprenorphine whereas most participants in our study agreed that it should be considered [18]. In our study, physicians reported more favorable attitudes with increased clinical education and exposure. In both studies, respondents agreed that EDinitiated buprenorphine was feasible if appropriate system supports were implemented.

Our findings align with previous research showing that emergency physicians perceive that system level facilitators including order sets and departmental protocols increase their uptake of procedures in the $\operatorname{ED}[16,25]$. Our recent Canada-wide survey of ED physicians also highlighted the potential value of order sets in shifting culture and practice [26]. Despite the availability of these resources, participants suggested that additional guidance around challenging cases would be helpful as part of the knowledge translation strategy, including complexities around polysubstance withdrawal, comorbidities, and relative contraindications to buprenorphine induction.

There is also a role for mentorship, continuing education, and additional clinical exposure. Our study found 
that a significant facilitator to adoption of buprenorphine in the ED was the presence of physician champions who led departmental change, consistent with literature which shows that role modeling builds physician comfort with new practices [27, 28]. Having outpatient clinics available addresses concerns of initiating a chronic medication in an acute setting, titrating the dose, and providing ongoing care. Rapid access clinics demonstrate positive clinical outcomes and retention in care, forming a crucial component of ED-initiated treatment for OUD [9, 29]. ED leadership should work closely with outpatient clinics to ensure rapid follow-up and a smooth transition of care from the ED to the outpatient clinic.

Our study identified persistent perceived barriers to buprenorphine use in an academic ED which had already initiated these supports including order sets, training, and follow up for patients. Additional barriers reported included lack of patient interest, discomfort with counselling around buprenorphine, lack of nursing support, time constraints and safety concerns as perceived barriers for ED physicians [16-18].. These findings highlight the need for earlier, more robust education for ED physicians and nurses on the use of buprenorphine.

Various frameworks suggest that guidelines are not adopted due to either knowledge, attitude or behaviours [30-34]. We found all three types of barriers in our findings; physicians reported lack of knowledge, uncertainty about the impacts of using buprenorphine for opioid withdrawal as well as environmental and patient factors as barriers to uptake. Treatment of OUD presents unique challenges in the ED given frequently associated social and medical complexities.

Additionally, the treatment of OUD is often stigmatized by physicians which can impact clinical outcomes $[35,36]$ and hinder knowledge translation [37]. Future studies should further explore the relationship between stigma and attitudes and perceived barriers to treating OUD.

Departments should develop a knowledge translation and implementation strategy that directly addresses perceived barriers in order to optimize guideline adoption [38]. Future research may also focus on understanding perceived barriers of health care administrators and allied health care staff in supporting buprenorphine prescribing in the ED setting [32]. These findings could inform our knowledge translation strategies, as we suspect from this study that early involvement of allied health professionals, including nursing and social work, in buprenorphine counselling would vastly improve uptake.

\section{Limitations}

This qualitative study had some important limitations. We interviewed physicians from one organization at one point in time which limits the generalizability of our findings. Generalizability is further limited by constructivist grounded theory methodology which considers the unique context and experiences of participants in shaping their reality which may vary considerably among emergency physicians. Although we randomly invited physicians, only half of physicians in the department were invited to participate to ensure that all interviews could be completed during the study period. Participants self-selected to participate in interviews which introduced some selection bias. Inviting all emergency physicians in the department could have improved sample variation. Finally, although participants were assured of confidentiality, we cannot exclude the possibility of socially desirable responses.

\section{Conclusion}

This study describes barriers and facilitators to addressing OUD and prescribing buprenorphine in a Canadian ED setting. Our findings suggest highly variable practices and the need for a targeted knowledge translation strategy to address common perceived barriers. Additional research is needed to elucidate perceived barriers of health care administrators and allied health care staff in supporting buprenorphine prescribing in the ED setting. Our findings suggest a role for physician champions, involvement of allied health professionals in counseling, and expanded education.

Abbreviations

OUD: opioid use disorder; ED: emergency department

\section{Supplementary Information}

The online version contains supplementary material available at https://doi. org/10.1186/s12873-021-00443-1.

Additional file 1.

Additional file 2.

\section{Acknowledgments \\ We would like to acknowledge Dr. Amy Gajaria who provided feedback and expertise which informed the final version of this article.}

\begin{abstract}
Authors' contributions
All authors contributed to the conceptualization and the design of the study. DW conducted the interviews. DW, PH and $\mathrm{JH}$ analyzed the interview transcripts. All authors contributed to data interpretation as well as authoring and revising the manuscript. The authors read and approved the final manuscript.
\end{abstract}

Funding

This work was supported by the University Health Network Emergency Medicine Research Fund and the University of Toronto Faculty of Medicine Comprehensive Research Experience for MD/PhD Students (CREMS) program. This research did not receive any other grants from funding agencies in the public, commercial, or not-forprofit sectors. 


\section{Availability of data and materials}

The datasets generated and/or analysed during the current study are not publicly available due to participant confidentiality but are available from the corresponding author on reasonable request.

\section{Declarations}

\section{Ethics approval and consent to participate}

The University Health Network Research Ethics Board approved the protocol as part of a quality improvement project. All methods were carried out in accordance with relevant guidelines and regulations. All participants provided informed consent after reviewing the study objectives. Participants were assured regarding confidentiality of participant identity and information.

\section{Consent for publication}

Not applicable.

\section{Competing interests}

The authors declare that they have no competing interests.

\section{Author details}

${ }^{1}$ Faculty of Medicine, University of Toronto, 1 King's College Circle, Toronto, Canada. ${ }^{2}$ Department of Family and Community Medicine, University of Toronto, Toronto, Canada. ${ }^{3}$ University Health Network Emergency Department, Toronto, Canada.

\section{Received: 17 December 2020 Accepted: 25 March 2021}

\section{Published online: 15 April 2021}

\section{References}

1. Hasin DS, O'Brien CP, Auriacombe M, Borges G, Bucholz K, Budney A, et al. DSM-5 criteria for substance use disorders: recommendations and rationale. Am J Psychiatr. 2013;170(8):834-51. https://doi.org/10.1176/appi.ajp.2013.12 060782.

2. Health Canada.Opioid-related harms in Canada: integrating emergency medical service, hospitalization, and death data. 2019. https://www.canada. ca/en/health-canada/services/substance-use/problematic-prescription-druguse/opioids/data-surveillance-research/integrating-emergency-medicalhospitalization-death-data.html. Accessed 16 Apr 2020

3. Bruneau J, Ahamad K, Goyer M-Ë, Poulin G, Selby P, Fischer B, et al. Management of opioid use disorders: a national clinical practice guideline. Can Med Assoc J. 2018;190(9):E247-57. https://doi.org/10.1503/cmaj.170958.

4. Kissin W, McLeod C, Sonnefeld J, Stanton A. Experiences of a national sample of qualified addiction specialists who have and have not prescribed buprenorphine for opioid dependence. J Addict Dis. 2006;25(4):91-103. https://doi.org/10.1300/J069v25n04_09.

5. West JC, Kosten TR, Wilk J, Svikis D, Triffleman E, Rae DS, et al. Challenges in Increasing Access to Buprenorphine Treatment for Opiate Addiction. Am J Addict. 2004;13(Suppl 1):S8-S16.

6. Ontario Drug Benefit Program. Ministry of Health and Long-Term Care.. 2021. https://www.ontario.ca/page/medication-coverage-results/

7. D'Onofrio G, O'Connor PG, Pantalon MV, Chawarski MC, Busch SH, Owens $\mathrm{PH}$, et al. Emergency department-initiated buprenorphine/naloxone treatment for opioid dependence: a randomized clinical trial. J Am Med Assoc. 2015;313(16):1636-44. https://doi.org/10.1001/jama.2015.3474.

8. D'Onofrio G, Chawarski MC, O'Connor PG, Pantalon MV, Busch SH, Owens $\mathrm{PH}$, et al. Emergency department-initiated buprenorphine for opioid dependence with continuation in primary care: outcomes during and after intervention. J Gen Intern Med. 2017;32(6):660-6. https://doi.org/10.1007/s11 606-017-3993-2

9. Wiercigroch D, Sheikh $H$, Hulme J. A rapid access to addiction medicine clinic facilitates treatment of substance use disorder and reduces substance use. Subst Abus Treat Prev Policy. 2020;15(1):4. https://doi.org/10.1186/s13 011-019-0250-1.

10. SchwarzR ZA, Bruce RD, Altice FL. Retention on buprenorphine treatment reduces emergency department utilization, but not hospitalization among treatment-seeking patients with opioid dependence. Subst Abus Treat. 2012:43(4):451-7. https://doi.org/10.1016/j.jsat.2012.03.008.

11. Squires DD, Gumbley SJ, Storti SA. Training substance abuse treatment organizations to adopt evidence-based practices: the addiction technology transfer Center of new England Science to service laboratory. J Subst Abus Treat. 2008;34(3):293-301. https://doi.org/10.1016/j.jsat.2007.04.010.

12. Hutchinson E, Catlin M, Andrilla CHA, Baldwin LM, Rosenblatt RA. Barriers to primary care physicians prescribing buprenorphine. Ann Fam Med. 2014; 12(2):128-33. https://doi.org/10.1370/afm.1595.

13. Netherland J, Botsko M, Egan JE, Saxon AJ, Cunningham CO, Finkelstein R, et al. Factors affecting willingness to provide buprenorphine treatment. I Subst Abus Treat. 2009;36(3):244-51. https://doi.org/10.1016/j.jsat.2008.06.006.

14. Cunningham CO, Sohler NL, McCoy K, Kunins HV. Attending physicians' and residents' attitudes and beliefs about prescribing buprenorphine at an urban teaching hospital. Fam Med. 2006;38(5):336-40.

15. Turner BJ, Laine C, Lin YT, Lynch K. Barriers and facilitators to primary care or human immunodeficiency virus clinics providing methadone or buprenorphine for the management of opioid dependence. Arch Intern Med. 2005;165(15):1769-76. https://doi.org/10.1001/archinte.165.15.1769.

16. Lowenstein M, Kilaru A, Perrone J, Hemmons J, Abdel-Rahman D, Meisel ZF, et al. Barriers and facilitators for emergency department initiation of buprenorphine: a physician survey. Am J Emerg Med. 2019;37(9):178717790. https://doi.org/10.1016/.j.ajem.2019.02.025.

17. Hawk KF, D'Onofrio G, Chawarski MC, O'Connor PG, Cowan E, Lyons MS, et al. Barriers and facilitators to clinician readiness to provide emergency department-initiated buprenorphine. JAMA. 2020;3(5):E204561.

18. Im D, Chary A, Condella A, Vongsachang $\mathrm{H}$, Carlson L. Emergency department clinicians' attitudes toward opioid use disorder and emergency department-initiated buprenorphine treatment: a mixed-methods study. West J Emerg Med. 2020;21(2):261-71. https://doi.org/10.5811/westjem.201 9.11.44382.

19. Charnaz K. Constructing grounded theory. 2nd ed. London: SAGE Publications; 2014

20. Glaser B, Strauss A. Discovery of grounded theory: strategies for qualitative research. New York: Walter de Gruyter; 1967.

21. Mills J, Bonner A, Francis K. The development of constructivist grounded theory. Int J Qual Methods. 2006;5(1):25-35. https://doi.org/10.1177/16094 0690600500103

22. Singh S, Estefan A. Selecting a grounded theory approach for nursing research. Glob Qual Nurs Res. 2018 Sep;1:5.

23. Patton M. Qualitative evaluation and research methods.2nd ed. Newbury Park: Sage Publications; 1990.

24. Priest KC, Gorfinkel L, Klimas J, Jones AA, Fairbairn N, Mccarty D. Comparing Canadian and United States opioid agonist therapy policies. Int J Drug Policy. 2019;74:257-65. https://doi.org/10.1016/j.drugpo.2019.01.020.

25. Kennedy J, Green RS, Stenstrom R. The use of induced hypothermia after cardiac arrest: A survey of Canadian emergency physicians. 2008;10(2):125-30.

26. Hoyeck P, Wiercigroch D, Clarke C, Moineddin R, Sheikh H, Hulme J. Understanding current practice of opioid use disorder management in emergency departments across Canada: a cross-sectional study. CJEM. 2020; 22(4):494-8. https://doi.org/10.1017/cem.2020.362.

27. Andrilla CHA, Moore TE, Patterson DG. Overcoming barriers to prescribing buprenorphine for the treatment of opioid use disorder: recommendations from rural physicians. J Rural Heal. 2019;35(1):113-21. https://doi.org/1 $0.1111 /$ jrh. 12328.

28. Huhn AS, Dunn KE. Why aren't physicians prescribing more buprenorphine? J Subst Abus Treat. 2017;78:1-7. https://doi.org/10.1016/j.jsat.2017.04.005.

29. Hu T, Snider-Adler M, Nijmeh L, Pyle A. Buprenorphine/naloxone induction in a Canadian emergency department with rapid access to communitybased addictions providers. CJEM. 2019;21(4):492-8 urnal_article.

30. Lang ES, Wyer PC, Haynes RB. Knowledge translation: closing the evidenceto-practice gap. Ann Emerg Med. 2007;49(3):355-63. https://doi.org/10.1016/ j.annemergmed.2006.08.022

31. Ebben RHA, Vloet LCM, Verhofstad MHJ, Meijer S, de Groot JAM, van Achterberg T. Adherence to guidelines and protocols in the prehospital and emergency care setting: a systematic review. Scand I Trauma Resusc Emerg Med. 2013;21(1):1.

32. Probst MA, Dayan PS, Raja AS, Slovis BH, Yadav K, Lam SH, et al. Knowledge translation and barriers to imaging optimization in the emergency department: a research agenda. Acad Emerg Med. 2015;22(12):1455-64. https://doi.org/10.1111/acem.12830.

33. Damschroder LJ, Aron DC, Keith RE, Kirsh SR, Alexander JA, Lowery JC. Fostering implementation of health services research findings into practice: a consolidated framework for advancing implementation science. Implement Sci. 2009;4(1):50. https://doi.org/10.1186/1748-5908-4-50. 
34. Michie S, Johnston M, Abraham C, Lawton R, Parker D, Walker A. Making psychological theory useful for implementing evidence based practice: a consensus approach. Qual Saf Heal Care. 2005;14(1):26-33. https://doi.org/1 0.1136/qshc.2004.011155.

35. Brener L, von Hippel W, von Hippel C, Resnick I, Treloar C. Perceptions of discriminatory treatment by staff as predictors of drug treatment

completion: utility of a mixed methods approach. Drug Alcohol Rev. 2010; 29(5):491-7. https://doi.org/10.1111/j.1465-3362.2010.00173.x.

36. Van Boekel LC, Brouwers EPM, Van Weeghel J, Garretsen HFL. Stigma among health professionals towards patients with substance use disorders and its consequences for healthcare delivery: systematic review. Drug Alcohol Depend. 2013;131(1-3):23-35. https://doi.org/10.1016/j.drugalcdep.2 013.02.018.

37. Olsen Y, Sharfstein JM. Confronting the stigma of opioid use disorder - and its treatment. JAMA. 2014;311(14):1393-4. https://doi.org/10.1001/jama.2 014.2147.

38. Rand CS, Powe NR, Wu AW, Wilson MH. Why don't physicians follow a framework for improvement. JAMA. 1999;282:1458-65.

\section{Publisher's Note}

Springer Nature remains neutral with regard to jurisdictional claims in published maps and institutional affiliations.

Ready to submit your research? Choose BMC and benefit from:

- fast, convenient online submission

- thorough peer review by experienced researchers in your field

- rapid publication on acceptance

- support for research data, including large and complex data types

- gold Open Access which fosters wider collaboration and increased citations

- maximum visibility for your research: over $100 \mathrm{M}$ website views per year

At BMC, research is always in progress.

Learn more biomedcentral.com/submissions 